02

\title{
Применение вейвлет-преобразований к анализу компонентов $2 D$-пика рамановского спектра трех- и четырехслойного графена
}

\author{
(C) Т.Е. Тимофреева, Е.П. Неустроев, В.И. Попов, П.В. Винокуров, В.Б. Тимофреев \\ Северо-восточный федеральный университет, \\ 677000 Якутск, Россия \\ e-mail: titamara2013@mail.ru
}

Поступила в редакцию 03.06.2018 г.

Предложен новый подход к анализу компонент $2 D$-пика рамановского спектра мультислойного графена, основанный на применении вейвлетов. В результате применения непрерывного вейвлет-преобразования визуализируются компоненты (подпики) $2 D$-пика рамановского спектра, определяются их число и частоты. Обнаружено, что для двухслойного и трехслойного графена с берналовской упаковкой (АВА) пик полосы $2 D$ рамановского спектра состоит из четырех и пяти компонент, соответственно. В случае четырехслойного графена с ромбоэдрической упаковкой $(\mathrm{ABC}) 2 D$-пик содержит пять компонент. Число детектируемых компонент и их частоты совпадают с экспериментальными данными.

DOI: $10.21883 /$ OS.2018.11.46815.153-18

\section{Введение}

Число слоев в мультиграфене и порядок их кристаллографической упаковки сильно влияют на электронные свойства, зонную структуру [1-17], электронфононное взаимодействие, фононную дисперсию $[18-$ 22 ], оптические свойства и химическую активность мультиграфена [23-27]. Спектроскопия комбинационного рассеяния света или рамановская спектроскопия является основным неразрушающим методом определения числа слоев мультиграфена, их относительной ориентации, совершенства кристаллической структуры графена. Она также широко применяется при исследовании влияния возмущающих факторов, таких как напряжение решетки, легирование и беспорядок, на свойства мультиграфена [28-36]. Рамановская спектроскопия также позволяет зондировать электронную структуру графена и электрон-фононное взаимодействие в графене. $2 D$-пик рамановского спектра мультиграфена изменяется по форме, ширине и положению с ростом числа слоев, отражая изменение зонной структуры.

В других работах $2 D$-пик обозначают как $G^{\prime}$ или $D^{*}, 2 D$ означает, что этот пик является обертоном пика $D$. Причиной возникновения $2 D$-пика является процесс междолинного рассеяния электрона на поперечном оптическом фононе (iТO), принадлежащем оптической ветви колебаний атомов углерода подрешеток А и В в плоскости графена в окрестности точки Дирака К. Из теории групп следует, что для двухслойного графена число разрешенных процессов двухрезонансного комбинационного рассеяния должно быть равным четырем. Для трехслойного графена вдоль линии Т $(\Gamma-\mathrm{K})$ зоны Бриллюэна могут быть реализованы пять процессов двухрезонансного комбинационного рассеяния с фононом $T^{+}$и четыре процесса с фононом $T^{-}$. Полное число возможных процессов двухрезонансного комбинационного рассеяния, предсказываемое теорией групп, равно пятнадцати [18]. Таким образом, согласно теории двухрезонансного рассеяния, должно быть четыре и девять оптических процессов, которые вносят вклад в полосу $2 D$ соответственно для двух- и трехслойного графена [37]. Однако, согласно работе [37], из-за вырождения средних компонент пики $2 D$ двух- и трехслойного графена содержат соответственно три и пять компонент. В других работах экспериментальные пики $2 D$ рамановских спектров двух- и трехслойного графена заполняются соответственно четырьмя и шестью лоренцевыми пиками с полушириной $24 \mathrm{~cm}^{-1}$ [38-42]. Таким образом, существует расхождение в определении числа лоренцевых компонент, вносящих вклад в $2 D$-пик трехслойного графена. В связи с этим представляет интерес проводить дальнейшее исследование структуры $2 D$-пика рамановского спектра мультиграфена для определения реального числа лоренцевых компонент, наблюдаемых в эксперименте.

В настоящей работе исследуются положения и число лоренцевых пиков полосы $2 D$ рамановских спектров двух-, трех-, четырехслойного графена на основе применения вейвлет-преобразования. Вейвлеты являются удобным, относительно недавно разработанным инструментом для обработки сигналов. Вейвлеты локализованы в физическом пространстве (во времени или в ,пространстве“) и в частотном пространстве, из них строят базисы анализирующих функций посредством переносов и масштабных преобразований для проведения вейвлетанализа. Вейвлет-анализ представляет собой разложение сигнала по этим анализирующим функциям и с точки зрения математики является вейвлет-преобразованием. Вейвлет-анализ - это математический „микроскоп“, позволяющий одновременно описать свойства быстро изменяющихся сигналов в разных временных масштабах. Вейвлет-анализ также успешно применяется для обработки изображений, распознавания речи и удаления шума из сигналов [43-46]. В данной работе применено 
непрерывное (интегральное) вейвлет-преобразование к рамановскому $2 D$-пику мультиграфена для того, чтобы визуализировать его составные компоненты (лоренцевы пики) и определить их число и частоты. Эти частоты являются фононными и соответствуют положениям максимумов лоренцевых пиков.

\section{Экспериментальная часть}

Чешуйки графена изготавливались методом микромеханического расслоения высокоориентированного пиролитического графита (ВОПГ) на подложке кремния с применением липкой голубой ленты (Nitto) и желтой ленты (kapton tape, DuPont). Использовали кремниевую подложку с толщиной оксидного слоя $\mathrm{SiO}_{2}$ в $300 \mathrm{~nm}$ для того, чтобы чешуйки графена были видимыми в оптическом микроскопе [47]. Предварительно подложки очищались от органических загрязнителей и часть подложек подвергались обработке кислородной плазмой. Число слоев микромеханически отслоенных чешуек графена можно оценить по оптическому изображению на подложке $\mathrm{SiO}_{2} / \mathrm{Si}$ (рис. 1). Число слоев мультиграфена и порядок их упаковки идентифицируются методом спектроскопии комбинационного рассеяния света или рамановской спектроскопии. Рамановские спектры снимались с помощью рамановского спектрометра (системы ИНТЕГРА СПЕКТРА, НТ-МДТ) с дифракционной решеткой, имеющей 600 штрихов на $\mathrm{mm}$ в диапазоне частот $400-3600 \mathrm{~cm}^{-1}$. Для проведения вейвлет-анализа полоса $2 D$ рамановского спектра снималась в диапазоне частот $2360-2950 \mathrm{~cm}^{-1}$ на дифракционной решетке 1800 штрихов на $\mathrm{mm}$. Образцы мультиграфена облучались лазером с длиной волны $532 \mathrm{~nm}(2.33 \mathrm{eV})$. Радиус пятна от лазерного пучка составлял $\sim 0.5 \mu \mathrm{m}$. Мощность лазера устанавливалась меньше $1 \mathrm{~mW}$, чтобы избежать перегрева поверхностей образцов мультиграфена. Изображения мультиграфена получались с помощью сканирующего электронного микроскопа (CЭM) (SEM, Jeol 7800 F).

Для того чтобы осуществить вейвлет-анализ полосы $2 D$ рамановского спектра, нами было использовано непрерывное (интегральное) вейвлет-преобразование

$$
C(a, b)=\frac{1}{\sqrt{a}} \int_{-\infty}^{\infty} \psi^{*}\left(\frac{t-b}{a}\right) s(t) d t,
$$

где $s(t)$ - анализируемый сигнал, $\psi^{*}-$ означает комплексно сопряженный вейвлет $\psi, b-$ параметр сдвига $\psi^{*}$. Параметр $a>0$ определяет размер анализирующего вейвлета и называется масштабным множителем (коэффициентом). Вейвлет растягивается, если $a<1$, и сжимается, если $a>1$. Результатом преобразования одномерной функции $s(t)$ является двумерная матрица амплитуд вейвлет-преобразования - значений коэффициентов $C(a, b)$, определенных в открытой $(b, a)$ полуплоскости $(b \in \mathbf{R}, a>0)$. Распределение этих значений в пространстве $(a, b)$ дает информацию об эволюции относительного вклада компонент сигнала разного временного (пространственного) масштаба. Каждая строка полученной матрицы $C(a, b)$ соответствует определенному значению параметра $a$ и представляет локальные особенности анализируемого сигнала при фиксированном масштабе $a$. В нашем случае $s(t)$ является пиковой кривой полосы $2 D$ рамановского спектра графена, а $t-$ частота $q(1 / \mathrm{cm})$ или так называемый рамановский сдвиг. Параметр $b$ задает положение локализации вейвлета в $q$.

Существует проблема, связанная с наличием шума у исходного сигнала $s$ полосы $2 D$ рамановского спектра мультиграфена. Перед применением вейвлетпреобразования шум был удален из анализируемого сигнала. Процедура очистки сигнала от шума осуществлялась с применением графического интерфейса пользователя Matlab (индивидуальная академическая лицензия № 1088874, MathWorks). Процедура очистки проводится на основе применения метода многоуровневого разложения и восстановления исходного сигнала без компонент, содержащих шум. Такой процесс включает в себя два аспекта: разложение сигнала для получения вейвлет-коэффициентов и восстановление сигнала из этих коэффициентов. Для того чтобы осуществить данную процедуру, были выбраны подходящий вейвлет и необходимое количество уровней разложения.

\section{Результаты и обсуждение}

Рамановские спектры чешуек мультиграфена накапливались с использованием лазера с длиной волны $532 \mathrm{~nm}$ $(2.33 \mathrm{eV}) .2 D$-пик рамановского спектра мультиграфена изменяет свою форму и ширину с изменением числа слоев, что позволяет отличить двухслойный графен от трехслойного [20,31,38]. Регистрация рамановских спектров в точках 1 и 6, 2, 5, 4 и 3 чешуйки мультиграфена $G 1$ (рис. $1, a$ ) показала пики $2 D$, характерные для одно-, двух-, трех-, четырех- и пятислойного графена (рис. 2). Рамановские спектры чешуйки мультиграфена $G 2$ (рис. $1, b)$ соответствуют монослойному (точка 1 ), двухслойному (точка 2) и трехслойному (точка 3 ) графену. Для того чтобы проверить число слоев $(n)$ графена, идентифицированное рамановской спектроскопией, была рассмотрена методика, предложенная в работе [48]. В этой работе предложен другой подход для подсчета числа слоев графена. Данная методика основана на измерении отношения интегральных интенсивностей $G$ пика графена и оптического фононного пика кремния $(\mathrm{Si})$ первого порядка, $I(G) / I(\mathrm{Si})$. Правильность результатов такого подхода была подтверждена измерениями толщины мультиграфена методом АCM и решением уравнений Френеля. Измерения были выполнены для графеновых чешуек, отложенных на подложках кремния с оксидным слоем $\mathrm{SiO}_{2}$ с толщиной 104 и $280 \mathrm{~nm}$, с использованием лазера с длиной волны $488 \mathrm{~nm}$ [48]. Сравнение данных для отношений $I(G) / I(\mathrm{Si})$, соответствующих числу слоев $n=1-5$, для двух подложек 


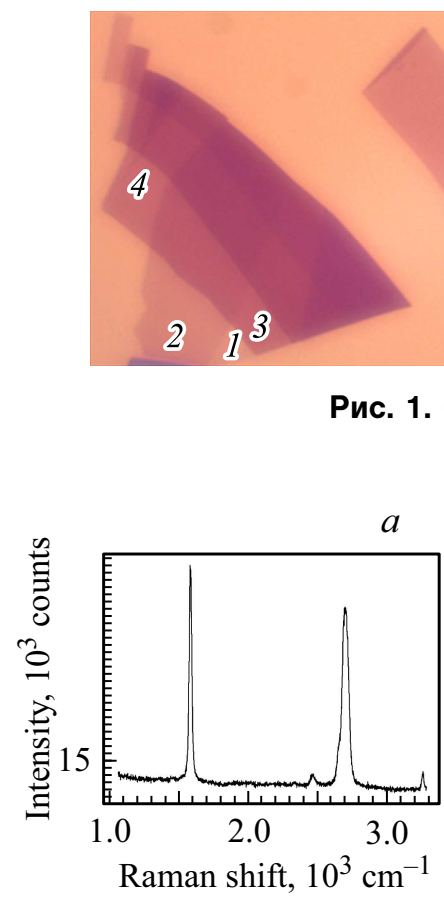

5

6
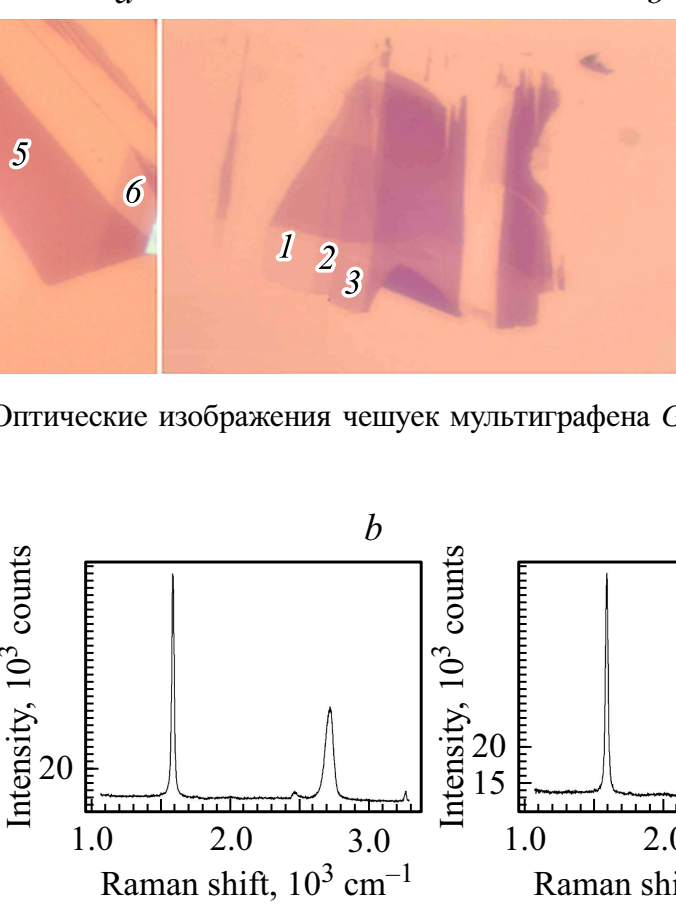

$b$

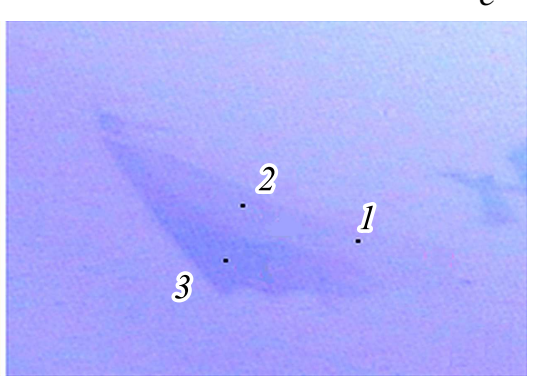

Рис. 1. Оптические изображения чешуек мультиграфена $G 1(a), G 2(b), G 3(c)$.
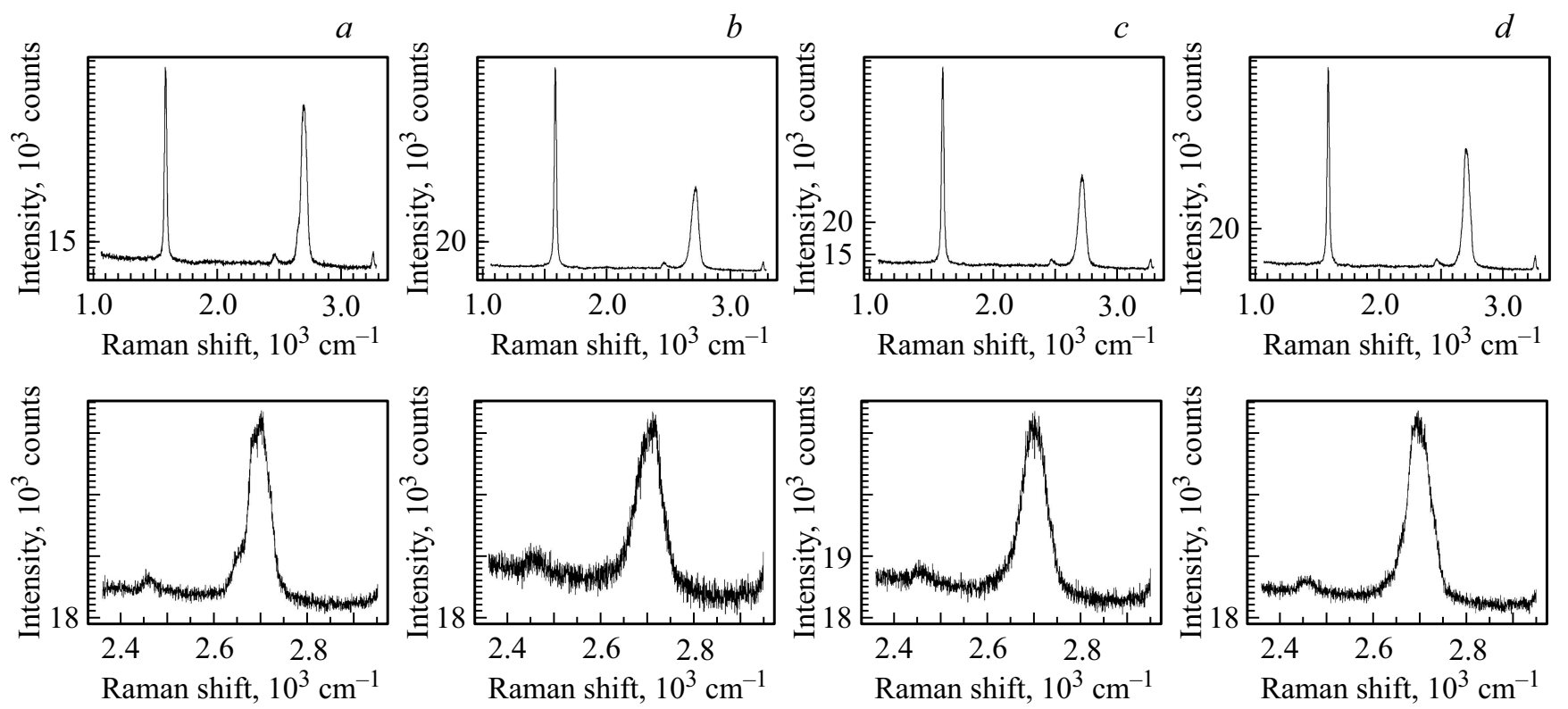

Рис. 2. $G$ - и $2 D$-пики рамановских спектров мультиграфена $G 1$, соответствующие точкам $2(a), 3(b), 4(c), 5(d)$. Энергия лазера $-2.33 \mathrm{eV}(\lambda=532 \mathrm{~nm})$.

показывает незначительное расхождение между ними, поэтому здесь эти отношения для оксидного слоя $\mathrm{SiO}_{2}$ c толщиной $280 \mathrm{~nm}$ могут быть использованы для подтверждения числа слоев, идентифицированных по $2 D$-пикам рамановских спектров.

Мы осушествили измерения отношений интегральных интенсивностей фононного пика кремния и $G$-пика мультиграфена $\left(I_{G} / I_{\mathrm{Si}}\right)$. Для сравнения полученных результатов с данными работы [48] значения последних были пересчитаны на длину волны лазера в $532 \mathrm{~nm}$ с использованием соотношений Френеля. Показатели преломления $\tilde{n}_{\mathrm{Gr}}=2.65-1.3 i, \tilde{n}_{\mathrm{Si}}=4.367-0.079 i$ для графена и кремния при $488 \mathrm{~nm}$ изменялись на значения $\tilde{n}_{\mathrm{Gr}}=2.67-1.34 i, \tilde{n}_{\mathrm{Si}}=4.152-0.0518 i$ при $532 \mathrm{~nm}$. Результаты измерений и перерасчетов отношений $I_{G} / I_{\mathrm{Si}}$ для графеновых чешуек образца $G 1$ с числом слоев от одного до пяти показаны в табл. 1. Обозначение $P n$ означает точку с номером $n$. Из табл. 1 можно увидеть, что пересчитанные отношения $I_{G} / I_{\mathrm{Si}}$ хорошо совпадают с результатами наших измерений.
Таблица 1. Отношения интегральных интенсивностей $G$-пика графена и оптического фононного пика кремния $(\mathrm{Si})$ первого порядка для чешуек мультиграфена $I(G) / I(\mathrm{Si})$

\begin{tabular}{c|c|c|c}
\hline $\begin{array}{c}\text { Число } \\
\text { слоев }\end{array}$ & $\begin{array}{c}\text { Наш эксперимент } \\
(\lambda=532 \mathrm{~nm})\end{array}$ & $\begin{array}{c}\text { Пересчитанные } \\
\text { данные }[48] \\
(\lambda=532 \mathrm{~nm})\end{array}$ & $\begin{array}{c}\text { Эксперимент [48] } \\
(\lambda=488 \mathrm{~nm})\end{array}$ \\
\hline 1 & $0.07(P 1)$ & 0.06 & 0.09 \\
2 & $0.12(P 2)$ & $0.11-0.12$ & $0.17-0.19$ \\
3 & $0.16(P 5, \mathrm{ABA})$ & 0.16 & 0.24 \\
4 & $0.2(P 4, \mathrm{ABCA})$ & 0.21 & 0.32 \\
5 & $0.31(P 3)$ & 0.29 & 0.44
\end{tabular}

Далее, попытались детектировать лоренцевы компоненты $2 D$-пиков рамановского спектра для двухи трехслойного графена. Экспериментальные спектры (пики 2D) обычно имеют шум. Перед применением вейвлет-преобразования шум был удален из сигнала $2 D$-пика рамановского спектра, основываясь на приме- 

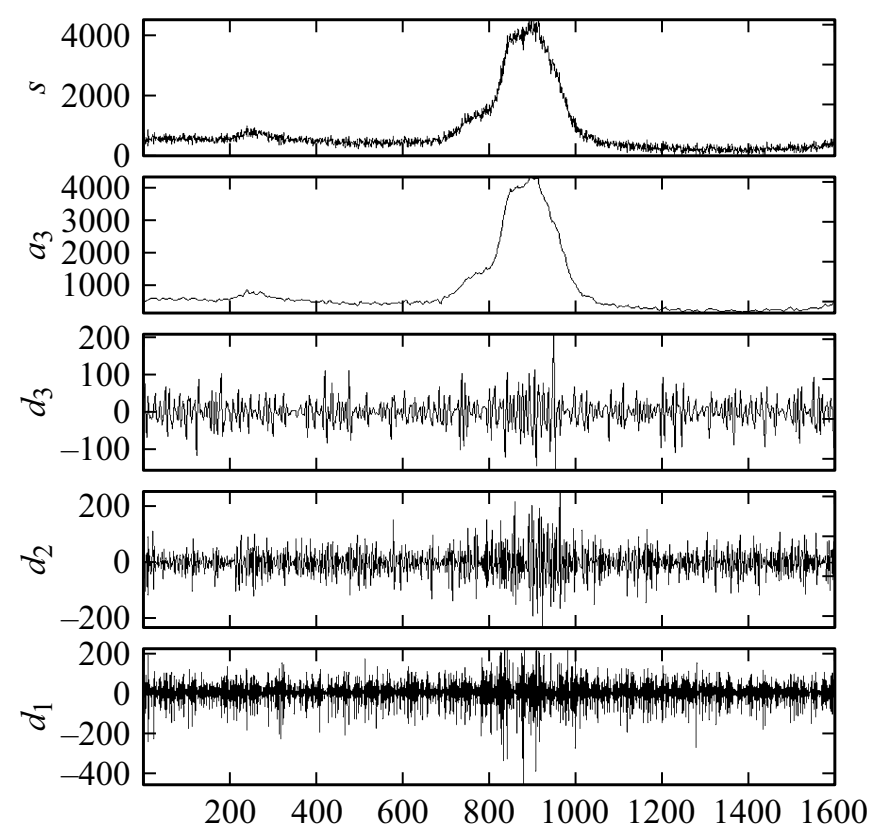

Рис. 3. Вейвлет-разложение $2 D$-пика рамановского спектра двухслойного графена (до уровня 3).

нении метода многоуровневого одномерного вейвлетанализа [43]. Очистка анализируемых сигналов от шума была осуществлена с использованием графического интерфейса MATLAB Wavelets Toolbox. На рис. 3 показано многоуровневое вейвлет-разложение сигнала (2D-пика двухслойного графена)

$$
s=a_{3}+d_{3}+d_{2}+d_{1}
$$

Соотношение (2) представляет трехуровневое вейвлетразложение сигнала $s$. Символы $d_{n}$ являются детализирующими коэффициентами $\left(c D_{n}\right)$ и содержат высокочастотные компоненты, соответствующие шумовой составляющей сигнала, в то время как аппроксимирующие коэффициенты $a_{n}\left(c A_{n}\right)$ содержат меньше шума, чем исходный сигнал. Из рис. 3 видно, что аппроксимирующий коэффициент $a_{n}$ почти не содержит шума по сравнению с исходным сигналом. Шум был удален из сигнала с использованием стратегии автоматической пороговой обработки. К детализирующим коэффициентам был применен мягкий тип пороговой обработки. После разложения и процедуры удаления шума из анализируемого одномерного сигнала, $2 D$-пика, было осуществлено восстановление сигнала без шумовых компонент. Выбор подходящего вейвлета и нужного уровня вейвлетразложения ведет к эффективной чистке сигнала от шума, что позволяет визуализировать пиковые компоненты $2 D$-пика и определить число слоев. В случае двухслойного графена $(P 2$, рис. 1$)$ удаление шума из рамановского $2 D$-пика осуществлялась с использованием вейвлетов Добеши „, $d b 3^{\text {“ } ~ и ~ в е и ̆ в л е т-р а з л о ж е н и я ~ д о ~ у р о в н я ~} 5$.

После процедуры удаления шума было осуществлено непрерывное вейвлет-преобразование эксперименталь-
Таблица 2. Частоты $\left(\mathrm{cm}^{-1}\right)$ лоренцевых пиков $P_{22}, P_{21}, P_{12}$, $P_{11}$ и $P_{1}$ полосы $2 D$ рамановского спектра двухслойного и однослойного графена

\begin{tabular}{c|c|c|c}
\hline Пик & {$[30,39]$} & $\begin{array}{c}a=13 \\
\text { (рис. } 1, b, P 2)\end{array}$ & $\begin{array}{c}a=12 \\
\text { (рис. } 1, a, P 2)\end{array}$ \\
\hline$P_{22}$ & 2650 & 2649 & 2647 \\
$P_{21}$ & 2681 & 2682 & 2679 \\
$P_{12}$ & 2700 & 2703 & 2701 \\
$P_{11}$ & 2716 & 2719 & 2719 \\
$P_{1}$ & $2675[30]$ & $2675(P 1)$ & $2674(P 1)$
\end{tabular}

ного пика рамановского спектра (рис. 1). Как упоминалось ранее, результатом вейвлет-преобразования является матрица коэффициентов $C(a, b)$ размерности $(a / \Delta a) \times 1024$, где $\Delta a$ является шагом изменения масштабного коэффициента $a$. Распределение значений этих коэффциентов в пространстве $(a, b)$ дает информацию о числе лоренцевых компонент $2 D$-пика и их частотах на разных масштабах. Для осуществления непрерывного вейвлет-преобразования экспериментального $2 D$-пика рамановского спектра мультиграфена был выбран гауссов вейвлет ,gaus2“. Масштабный коэффициент $a$ задавался от 1 до 20 с шагом 0.2. График коэффициентов строки показывает локализацию лоренцевых пиков $q$ $(1 / \mathrm{cm})$ при фиксированном значении масштабного коэффициента $a$. Значение масштабного параметра „а“ оценили согласно соотношению

$$
a=\frac{w_{2 D}}{T \Delta q},
$$

где $w_{2 D}-$ полуширина полосы $2 D$ рамановского спектра, $T$ - носитель вейвлета, $\Delta q=0.367 \mathrm{~cm}^{-1} /$ graduation - интервал дискретизации $q(1 / \mathrm{cm})$, $\Delta q=\left(q_{2}-q_{1}\right) / 1600$, где $q_{2}, q_{1}$ - конечная и начальная точка оси рамановского сдвига. Эта формула дает оценку сверху значения параметра $a$. Реальное значение масштабного коэффциента $a$ может быть несколько ниже этого значения из-за необходимости разрешения пиковых компонент.

Результаты вейвлет-преобразования показывают, что $2 D$-пик двухслойного графена содержит четыре лоренцевых пика, обозначаемые как $P_{i j}(i, j=1,2)$ [49]. Каждый пик соответствует одному из процессов двухрезонансного комбинационного рассеяния. Пик с наибольшей частотой связан с процессом $P_{11}$, который соответствует максимальному значению $q\left(q_{11}\right)$. Наименьшее значение $q\left(q_{22}\right)$ связано с процессом $P_{22}$, с которым связана наименьшая пиковая частота $q(1 / \mathrm{cm})$ полосы $2 D$. Два промежуточных пика связаны с процессами $P_{12}$ и $P_{21}$ [37,39]. В настоящей работе также показано, что существуют четыре пика для двухслойного графена. На рис. 4, $a$ показаны анализируемый сигнал $s$ (пик полосы $2 D$ двухслойного графена) и его образ, очищенный от шума. Коэффициенты непрерывного вейвлет- 

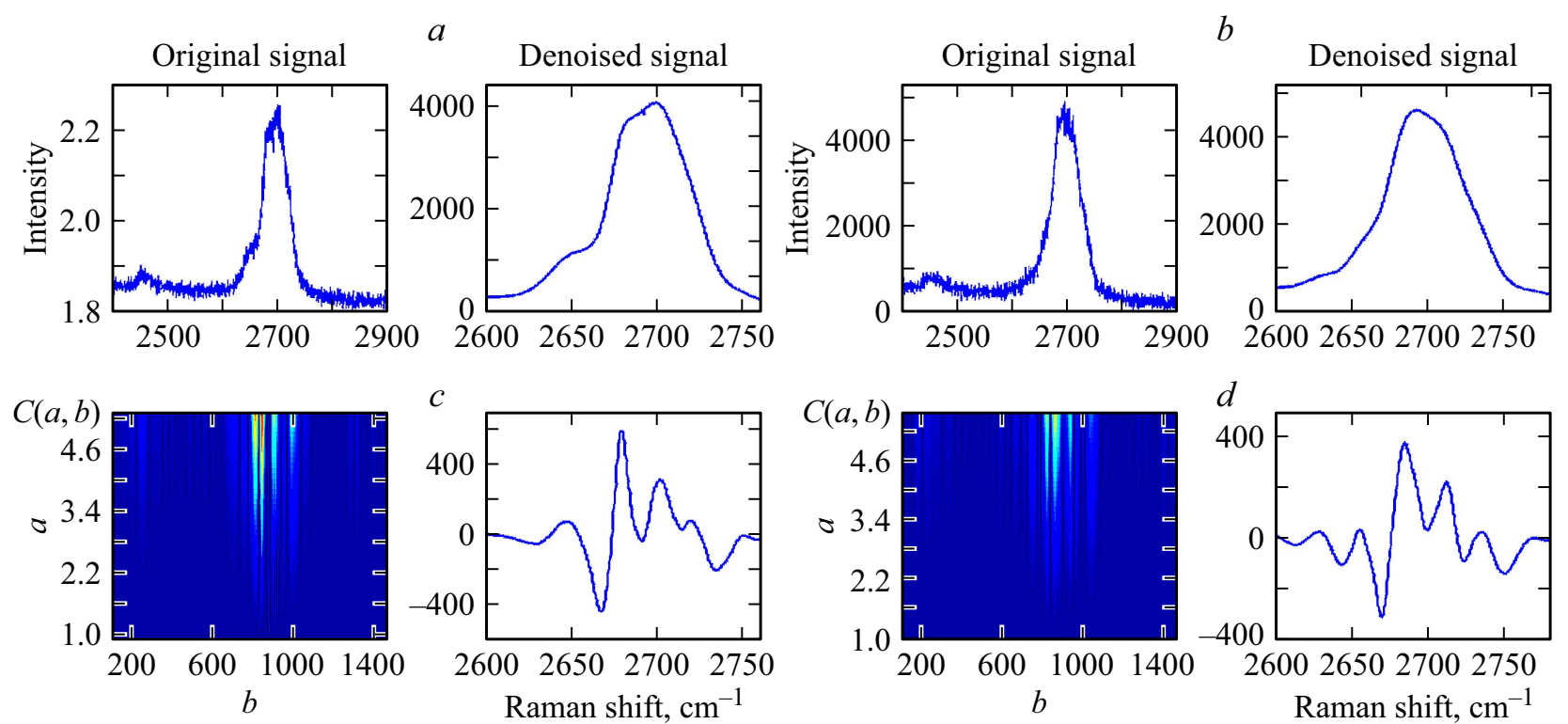

Рис. 4. $(a, b)$ Исходный и очищенный от шума сигналы $(2 D$-пик рамановского спектра), $(c, d)$ вейвлет-коэффициенты $C$ (вейвлетспектр) и график $C$ при фиксированном значении масштабного коэффициента $a=12$ для двух- $(a, c)$ и трехслойного графена с берналовской упаковкой $(b, d)$ при $a=15$.
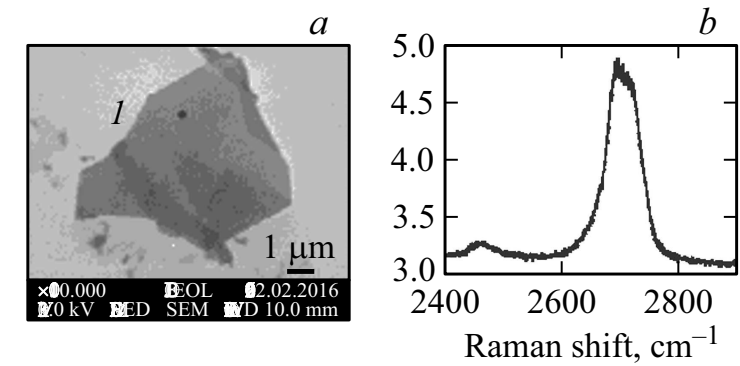
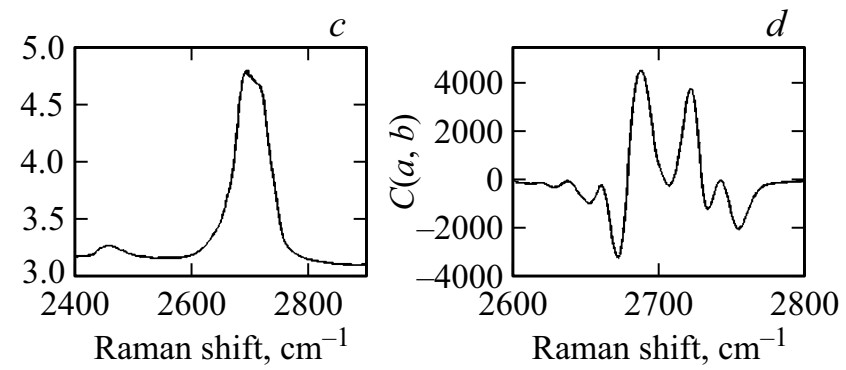

Рис. 5. СЭМ-изображение $(a)$, исходный $(b)$ и очищенный $(c)$ сигналы (2D-пик) четырехслойного графена с упаковкой $\mathrm{ABC}(G 4)$, вейвлет-спектр коэффициентов $C(a, b)$ при $a=15(d)$.

преобразования $C(a, b)$ представляют степень корреляции вейвлета с анализируемой частью сигнала. Чем больше $C$, тем больше подобие между вейвлетом и сигналом. Ниже на рис. 4, $c$ можно увидеть картину коэффициентов $C$ или вейвлет-спектр $2 D$-пика, очищенный от шума, и график коэффициентов при $a=12$ с четырьмя пиками. Частоты четырех обнаруженных лоренцевых пиков приведены в табл. 2. Полученные значения частот хорошо согласуются с данными работ [30,39].

На рис. $4, b$ представлены исходный и очищенный от шума $2 D$-пик рамановского спектра трехслойного графена с берналовской упаковкой (ABA), у которого число слоев и тип упаковки также были идентифицированы по отношению $I_{G} / I_{\mathrm{Si}}$ (табл. 1) и полуширине пика. Согласно литературным данным, полоса $2 D$ трехслойного графена содержит шесть компонент (лоренцевых пиков) с полушириной $\sim 24 \mathrm{~cm}^{-1}[13,30,39,41]$. Наши результаты (рис. $4, d$ ) показывают пять компонент. Это число подтверждается экспериментальными данными и теоретическим предсказанием, полученным на осно- ве расчета интенсивности рамановского спектра [37]. Частоты полученных компонент хорошо согласуются с данными работы [41] (табл. 3). Пик при частоте $\sim 2700 \mathrm{~cm}^{-1}$ отсутствует. Значения фононных частот полученных компонент приведены в табл. 3.

Далее были зарегистрированы рамановские спектры образца мультиграфена (G4) и был обнаружен четрырехслойный графен с ромбоэдрической упаковкой (ABC) (рис. 5,a). Четырехслойный графен с упаковкой АВС имеет более широкий и более асимметричный пик $2 D$ в сравнении с трехслойным графеном с упаковкой ABА. Действительно, полуширина пика трехслойного графена с берналовской (AВА) упаковкой составляет $59.5 \mathrm{~cm}^{-1}$, а четырехслойного графена $\mathrm{ABC}-63.3 \mathrm{~cm}^{-1}$. Число слоев идентифицировалось по отношению $I_{G} / I_{\mathrm{Si}}=0.21$ (табл. 1) и по полуширине пика [42]. Непрерывное вейвлет-преобразование показывает, что $2 D$-пик содержит пять составных пиков (рис. 5, d). Частоты последних приведены в табл. 3, столбец 7. Сравнение результатов непрерывного вейвлет-преобразования с экспери- 
Таблица 3. Частоты полос $2 D$ рамановского спектра мультиграфена $\left(\mathrm{cm}^{-1}\right)$

\begin{tabular}{c|c|c|c|c|c|c}
\hline AВА [41] & {$[37]$} & $\begin{array}{c}\text { АВА вейвлет-анализ } \\
\text { (рис. } 1, b, P 3)\end{array}$ & $\begin{array}{c}\text { АВА вейвлет-анализ } \\
\text { (рис. 1, } c, P 2)\end{array}$ & $\begin{array}{c}\text { АВА вейвлет-анализ } \\
\text { (рис. 1, } a, P 5)\end{array}$ & $\begin{array}{c}\text { АВС }[41] \\
\text { АВСА вейвлет-анализ } \\
(\text { рис. 5, } a, P 1)\end{array}$ \\
\hline 2625 & - & 2625 & 2628 & 2630 & - & \\
- & - & - & - & - & 2634 & 2638 \\
2663 & $2652 \pm 1$ & 2658 & 2661 & 2652 & 2653 & 2660 \\
2693 & $2673 \pm 1$ & 2682 & 2691 & - & 2684 & 2689 \\
2700 & $2699 \pm 1$ & - & - & 2711 & 2719 & - \\
2713 & $2718 \pm 1$ & 2715 & 2716 & 2732 & 2734 & 272 \\
2735 & $2737 \pm 1$ & 2738 & 2739 & &
\end{tabular}

ментальными данными [37,41] для трехслойного графена с АВС-упаковкой показывает отсутствие компоненты пика при фононной частоте $2700 \mathrm{~cm}^{-1}$. Более узкая полоса частот наблюдается у трехслойного графена с АВС-упаковкой на подложке, обработанной кислородной плазмой (табл. 3, столбец 5).

Также был рассмотрен шестислойный графен с берналовской упаковкой $\left(I_{G} / I_{\mathrm{Si}}=0.31\right)$ с полушириной пика $w_{2 D}=63.7 \mathrm{~cm}^{-1}$. Вейвлет-анализ показывает следующие результаты - число компонент 5, частоты пиков $2625,2653,2684,2711,2731 \mathrm{~cm}^{-1}$.

\section{Заключение}

Предложен подход к анализу структуры $2 D$-пика мультиграфена, основанный на применении вейвлетов. Мы зарегистрировали и проанализировали рамановские спектры графена с числом слоев от одного до четырех на кремниевой подложке с оксидным слоем с толщиной $300 \mathrm{~nm}$ с использованием лазера с длиной волны $532 \mathrm{~nm}(2.33 \mathrm{eV})$. Число слоев мультиграфена было идентифицировано по отношению интегральных интенсивностей $G$-пика графена и оптического фононного пика кремния $(\mathrm{Si})$ первого порядка $\left(I_{G} / I_{\mathrm{Si}}\right)$, а также по оптическому изображению. Непрерывное вейвлетпреобразование сигнала (2D-пика мультиграфена) позволяет визуализировать компоненты $2 D$-пика и определить их число и частоты. Перед применением непрерывного вейвлет-преобразования сигнал был очищен от шума с использованием подходящего вейвлета и необходимого количества уровней вейвлет-разложения сигнала. Значение масштабного коэффициента „а“ было оценено по формуле, предложенной в этой работе. Эффективное удаления шума из сигнала и выбор масштабного коэффициента „а“ являются решающими факторами для корректного определения числа компонент пика $2 D$. Число и значения частот компонент $2 D$-пика рамановского спектра двухслойного графена подтверждают теоретические и экспериментальные данные. Обнаружено, что у трехслойного графена с берналовской упаковкой существуют пять компонент $2 D$-пика в согласии с предсказанием расширенного метода сильной связи и с экспериментом J. Park, M. Dresselhaus [37].
Согласно данным непрерывного вейвлет-преобразования полоса $2 D$ четырехслойного графена с ромбоэдрической упаковкой также содержит пять пиков. Данный подход, основанный на применении вейвлет-анализа, может быть применен к рамановским спектрам других двумерных материалов и к спектрам другой природы, в частности, к рентгеновским фотоэлектронным спектрам.

Авторы выражают благодарность Северо-Восточному Федеральному университету за финансовую поддержку, проф. Е.И. Михайловой. Авторы также хотели бы поблагодарить С.А. Смагулову за поддержку этой работы.

\section{Список литературы}

[1] Mak K.F., Shan J., Heinz T.F. // Phys. Rev. Lett. 2010. V. 104. P. 176404 (4). doi 10.1103/PhysRevLett.104.176404

[2] Guinea F., Castro Neto A.H., Peres N.M.R. // Solid State Commun. 2007. V. 143. P. 116. doi 10.1016/j.ssc.2007.03.053

[3] Guinea F., Castro Neto A.H., Peres N.M.R. // Phys. Rev. B. 2006. V. 73. P. 245426. doi 10.1103/PhysRevB.73.245426

[4] Lui C.H., Li Zh., Chen Zh., Klimov P.V., Brus L.E., Heinz T.F. // Nano Lett. 2011. V. 11. P. 164. doi 10.1021/n11032827

[5] Aoki M.H., Amawashi H. // Sol. State Comm. 2007. V. 142. P. 123. doi 10.1016/j.ssc.2007.02.013

[6] Tabert C.J., Nicol E.J. // Phys. Rev. B. 2012. V. 86. P. 075439. doi 10.1103/PhysRevB.86.075439

[7] Koshino M., McCann E. // Phys. Rev. B. 2013. V. 87. P. 45420. doi 10.1103/PhysRevB.87.045420

[8] Zhang F., Sahu B., Min H., MacDonald A.H. // Phys. Rev. B. 2010. V. 82. P. 035409. doi 10.1103/PhysRevB.82.035409

[9] Bao C., Yao W., Wang E., Chen C., Avila J., Asensio M.C. et al. // Nano Lett. 2017. V. 17. P. 1564.

doi 10.1021/acs.nanolett.6b04698

[10] Craciun M.F., Russo S., Yamamoto M., Oostinga J.B., Morpurgo A.F. and Tarusha S. // Nature Nanotechnology. 2009. V. 4. P. 383. doi 10.1038/nnano.2009.89

[11] Bao W., Jing L., Velasco Jr., Lee Y., Liu G., Tran D., et al. // Nature Phys. 2011. V. 7. P. 948. doi 10.1038/nphys 2103

[12] Avetisyan A.A., Partoens B., Peeters F.M. // Phys. Rev. B. 2010. V. 81. P. 115432. doi 10.1103/PhysRevB.81.115432

[13] Khodkov T., Khrapach I., Craciun M.F., Russo S. // Nano Lett. 2015. V. 15. P. 4429. doi 0.1021/acs.nanolett.5b00772 
[14] Grushina A.L., Ki D-K., Koshino M., Nicolet A.A.L., Faugeras C., McCann E. et al. // Nat. Commun. 2015. V. 6. P. 6419. doi $10.1038 /$ ncomms 7419

[15] Nam Y., Ki D.-K., Koshino M., McCann E., Morpurgo A.F. // 2D Materials. 2016. V. 3. P. 045014.

[16] Koshino M., Sugisawa K., McCann E. // Phys. Rev. B. 2017. V. 95. P. 235311. doi 10.1103/PhysRevB.95.235311

[17] Myhro K., Che S., Shi Y., Lee Y., Thilahar K., Bleich K. et al. Электронный ресурс. Код доступа: arXiv:1803.03222. 2018.

[18] Malard L.M., Guimaraes M.H.D., Mafra D.L., Mazzoni M.S.C., Jorio A. // Phys. Rev. B. 2009. V. 79. P. 125426. doi 10.1103/PhysRevB.79.125426

[19] Saha S.K., Waghmare U.V., Krishnamurthy H.R., Sood A.K. // Phys. Rev. B. 2008. V. 78. P. 165421. doi 10.1103/PhysRevB.78.165421

[20] Gupta A., Chen G., Joshi P., Tadigadapa S., Eklund P. // Nano Lett. 2006. V. 6. P. 2767. doi 10.1021/n1061420a

[21] Jiang J.W., Tang H., Wang B.S., Su Z.B. // Phys. Rev. B. 2008. V. 77. P. 235421. doi 10.1103/PhysRevB.77.235421

[22] Yan J., Zhang Y., Kim P., Pinczuk A. // Phys. Rev. Lett. 2007. V. 98. P. 166802. doi 10.1103/PhysRevLett.98.166802

[23] Lui C.H., Cappelluti E., Li Zh., and Heinz T.F. // Phys. Rev. Lett. 2013. V. 110. P. 185504. doi 10.1103/PhysRevLett.110.185504.

[24] Mak K.F., Sfeir M.Y., Misewich J.A., Heinz T.F. // Proc. Natl. Acad. Sci. 2010. V. 107. P. 14999. doi 10.1073/pnas.1004595107

[25] Havener R.W., Liang Y., Brown L., Yang L., Park J. // Nano Lett. 2014. V. 14. P. 3353. doi 10.1021/n1500823k

[26] Patel H., Havener R.W., Brown L., Liang Y., Yang L., Park J. et al. // Nano Lett. 2015. V. 15. P. 5932. doi 10.1021/acs.nanolett.5b02035

[27] Sharma R., Baik J.H., Perera C.J., Strano M.S. // Nano Lett. 2010. V. 10. P. 398. doi 10.1021/n1902741x

[28] Ferrari A.C, Basko D.M. // Nature nanotechnology. 2013. V. 8. P. 235. doi 10.1038/nnano.2013.46

[29] Ferrari A.C. // Solid State Comm. 2007. V. 143. P. 47. doi 10.1016/j.ssc.2007.03.052

[30] Malard L.M., Nilsson J., Elias D.C., Brant J.C, Plentz F., Alved E.S. et al. // Phys. Rev. B. 2007. V. 76. P. 201401. doi 10.1103/PhysRevB.76.201401

[31] Graf D., Molitor F., Ensslin K., Stampfer C., Jungen A., Hierold C. et al. // Nano Lett. 2007. V. 7. P. 238. doi $10.1021 / \mathrm{n} 1061702 \mathrm{a}$

[32] Das A., Chakraborty B., Sood A.K. // Bull. Mater. Sci. 2008. V. 31. P. 579. doi 10.1007/s12034-008-0090-5

[33] Bayle M., Reckinger N., Felten A., Landois P., Lancry O., Dutertre B. et al. // J. Raman Spectrosc. 2018. V. 49. P. 36. doi $10.1002 /$ jrs.5279

[34] Yoon D., Moon H., Cheong H., Choi J., Choi J., Park B. // J. Korean Phys. Soc. 2009. V. 55. P. 1299. doi $10.3938 / \mathrm{jkps} .55 .1299$

[35] Rao R., Podila R., Tsuchikawa R., Katoch J. et al. // ACS Nano. 2011. V. 5. P. 1594. doi 10.1021/nn1031017

[36] Herziger F., Tyborski C., Ochedowski O., Schleberger M., Maultzsch J. // Carbon. 2018. V. 133. P. 254. doi 10.1016/j.carbon.2018.03.026

[37] Park J.S., Reina A., Saito R., Kong J., Dresselhausd G., Dresselhaus M.S. // Carbon. 2009. V. 47. P. 1303. doi 10.1016/j.carbon.2009.01.009
[38] Ferrari A.C., Meyer J.C., Scardaci V., Casiraghi C., Lazzeri M., Mauri F. et al. // Phys. Rev. Lett. 2006. V. 97. P. 187401. doi 10.1103/physrevlett.97.187401

[39] Malard L.M., Pimenta M.A., Dresselhaus G., Dresselhaus M.S. // Phys. Rep. 2009. V. 473. P. 51. doi 10.1016/j.physrep.2009.02.003

[40] Ni Z., Wang Y., Yu T., Shen Z. // Nano Research. 2008. V. 1. P. 273. doi 10.1007/s12274-008-8036-1

[41] Cong C., Yu T., Sato K., Shang J., Saito R., Dresselhaus G.F., Dresselhaus M.S. // ACS Nano. 2011. V. 5. P. 8760. doi 10.1021/nn203472f

[42] Hao Y., Wang Y., Wang L., Ni Z., Wang Z., Wang R. et al. // Small. 2010. V. 6. P. 195. doi 10.1002/smll.200901173

[43] Смоленцев Н.К. Основы теории вейлетов. Вейвлеты в MATLAB. М.: ДМК, 2005. 303 c.

[44] Acтафбева H.М. // УФН. 1996. Т. 166. № 11. С. 1145.; Astafieva N.M. // Phys. Usp. 1996. V. 39. P. 1085. doi 10.3367/UFNr.0166.199611a.1145.

[45] Rioul O., Vetterli M. // IEEE Signal Processing Magazine. 1991. V. 8. P. 14. doi 10.1109/79.91217

[46] Misiti M., Misiti Y., Oppenheim G., Poggi J.M. Wavelets and Their Applications.London: ISTE, 2007. doi 10.1002/9780470612491.fmatter

[47] Blake P., Hill E.W., Castro Neto A.H., Novoselov K.S., Jiang D., Yang R. et al. // Appl. Phys. Lett. 2007. V. 91. P. 063124. doi 10.1063/1.2768624

[48] Koh Y.K., Bae M.-H., Cahill D.G., Pop E. // ACS Nano. 2011. V. 5. P. 269. doi $10.1021 / \mathrm{nn} 102658 \mathrm{a}$

[49] Тимофбеева Т.Е., Смагулова С.А., Попов В.И. // ФТП. 2015. T. 49. B. 6. C. 834. Timofeeva T.E., Smagulova S.A., Popov V.I. // Semiconductors. 2015. V. 49. P. 834. doi 10.1134/S1063782615060251 